\title{
Dynamics of shallow dark solitons in a trapped gas of impenetrable bosons
}

\author{
D.J. Frantzeskakis ${ }^{1}$, N.P. Proukakis ${ }^{2}$ and P.G. Kevrekidis ${ }^{3}$ \\ ${ }^{1}$ Department of Physics, University of Athens, Panepistimiopolis, Zografos, Athens 157 84, Greece \\ 2 Department of Physics, University of Durham, South Road, Durham DH1 3LE, United Kingdom \\ 3 Department of Mathematics and Statistics, University of Massachusetts, Amherst MA 01003-4515, USA
}

\begin{abstract}
The dynamics of linear and nonlinear excitations in a Bose gas in the Tonks-Girardeau (TG) regime with longitudinal confinement are studied within a mean field theory of quintic nonlinearity. A reductive perturbation method is used to demonstrate that the dynamics of shallow dark solitons, in the presence of an external potential, can effectively be described by a variable-coefficient Korteweg-de Vries equation. The soliton oscillation frequency is analytically obtained to be equal to the axial trap frequency, in agreement with numerical predictions obtained by Busch et al. [J. Phys. B 36, 2553 (2003)] via the Bose-Fermi mapping. We obtain analytical expressions for the evolution of both soliton and emitted radiation (sound) profiles.
\end{abstract}

Dark solitons are fundamental nonlinear excitations of the nonlinear Schrödinger (NLS) equation with repulsive interactions, which have attracted much attention in the field of atomic Bose-Einstein condensates (BECs), where they have been studied experimentally [1] and theoretically in the framework of the Gross-Pitaevskii (GP) equation [2-9]. In particular, under conditions of tight transverse confinement, for which the gas becomes quasione-dimensional, dark solitons in longitudinal harmonic traps have been shown to oscillate with frequency $\Omega / \sqrt{2}$, where $\Omega$ is the axial trap frequency [2-4]. Thermal [5] and dynamical [6] instabilities, including sound emission phenomena $[7,8]$, have also been investigated. Furthermore, apart from the traditional rectilinear solitons, ringshaped dark solitons, have also been proposed [9].

For very tight transverse confinement and sufficiently low densities $(n \ll 1 / \xi$, where $\xi$ the healing length of the system) [10], an atomic gas with repulsive interactions enters the Tonks-Girardeau (TG) regime [11], where it behaves like a one-dimensional gas of impenetrable bosons. This regime, which is currently under intense experimental investigation [12], has attracted considerable theoretical attention both in the absence [13-15], as well as in the presence of axial confinement [16-18]. Dark solitons have also been predicted to occur in the TG regime, either upon employing the Bose-Fermi mapping theorem $[14,19]$, or via a mean-field approach of quintic nonlinearity arising in this limit [20,21] (see also relevant work for fermionic systems [22]). However, some important issues of soliton dynamics, such as its instability to sound emission due to the longitudinal confinement, and the profiles of the emitted sound waves have not been discussed in detail. It is the aim of this paper to address these points analytically in the case of shallow solitons.

Our analysis is based on the modified NLS equation with a quintic nonlinearity (as opposed to the cubic nonlinear GP equation valid in 3D systems [23]) given by

$$
i \hbar \frac{\partial \psi}{\partial t}=\left[-\frac{\hbar^{2}}{2 m} \frac{\partial^{2}}{\partial x^{2}}+\frac{\pi^{2} \hbar^{2}}{2 m}|\psi|^{4}+\frac{1}{2} m \omega_{x}^{2} x^{2}\right] \psi
$$

Here $\psi$ is the order parameter of the system (normalized to the number of particles $N), m$ the atomic mass and $\omega_{x}$ the axial confining frequency. The above long wavelength equation [20], originally derived in [24] from a renormalization group approach, has also been obtained by other techniques, such as (i) the Kohn-Sham density functional theory for bosons $[21,25,26]$, (ii) an in-depth study of the energy dependence of the many-body effective interaction [27], (iii) a rigorous analysis of the many-body Schrödinger equation [28]. This equation is expected to be valid for weak density modulations, as long as the number of atoms exceeds a certain minimum value (typically much larger than 10), for which oscillations in the density profiles become essentially suppressed $[20,27,29]$. Note that an alternative density-functional approach to deal with the excitations and dynamics in the TG limit has been recently proposed [30].

The validity of Eq. (1) has been criticized in certain regimes where the outcome is sensitive to the exact phase of the order parameter, e.g., interference experiments on a torus, where effects beyond the realm of mean field theory arise, and for which Eq. (1) overestimates the coherence properties [31]. The latter treatment, however, ignores longitudinal confinement which is the key source of the dynamics studied in this paper. An alternative exact treatment [19] including the harmonic confinement has predicted soliton oscillations at the trap frequency (this result was explicitly demonstrated numerically for deep dark solitons). This paper complements the result of [19], in that the same oscillation frequency is obtained analytically for shallow dark solitons, for which the employed hydrodynamic approximation is expected to be valid.

Solitons moving on a background density gradient are prone to sound emission $[2-4,8]$, but are stabilized against decay in a single harmonic trap by the continuous sound emission-reabsorption cycles [7]. We derive analytical results for both the soliton amplitude and speed as well as associated sound profiles. Starting from Eq. (1), our analytical predictions are obtained by means of a reductive multi-scale perturbative method, which yields a variable coefficient Korteweg-de Vries (KdV) equation for the dynamics of shallow dark solitons. Note that the 
above quintic NLS equation is a model of mathematical interest in its own right, as "traditional" analytical techniques, such as the adiabatic perturbation theory for dark solitons in BECs [4,9], cannot be applied for such nonlinearities. However, a similar technique as the one presented here has been succesfully applied to quasi-onedimensional condensates in the GP limit [3]. To start our analysis, we express Eq. (1) in dimensionless form,

$$
i u_{t}=-\frac{1}{2} u_{x x}+|u|^{4} u+V(x) u,
$$

where the subscripts denote partial differentiation, while the variables $t, x$ and the function $u$ are measured, respectively, in units of $m /\left(\hbar \pi^{2} n_{\mathrm{o}}^{2}\right), 1 /\left(\pi n_{\mathrm{o}}\right)$ and $2^{1 / 4} \sqrt{n_{\mathrm{o}}}$ (where $n_{\mathrm{o}} \equiv\left|\psi_{\mathrm{o}}\right|^{2}$ the peak density of the gas). The external potential in Eq. (2) is $V(x)=(1 / 2)\left(\pi n_{\mathrm{o}} a_{x}\right)^{-4} x^{2}$, where $a_{x} \equiv \sqrt{\hbar /\left(m \omega_{x}\right)}$ is the harmonic oscillator length in the axial direction. Taking into regard that the parameter $\left(\pi n_{\mathrm{o}} a_{x}\right)^{-4}$ is apparently small, it is convenient to define the small parameter $\epsilon \equiv \Omega^{-2 / 3}\left(\pi n_{\mathrm{o}} a_{x}\right)^{-4 / 3}[\Omega$ is a parameter of order $O(1)$ ], which will be used in the perturbation analysis to be presented below. This way, the external potential takes the form $V(X)=(1 / 2) \Omega^{2} X^{2}$, i.e., it is a function of the slow variable $X=\epsilon^{3 / 2} x$, while $\Omega$ expresses the strength of the magnetic trap, or the normalized axial trap frequency. By analogy to [29], we introduce the Madelung transformation $u=\sqrt{n} \exp (i \phi)$ ( $n$ and $\phi$ denote the density and phase respectively) to reduce Eq. (2) to the following hydrodynamic equations,

$$
\begin{aligned}
& n_{t}+\left(n \phi_{x}\right)_{x}=0, \\
& \phi_{t}+\frac{1}{2} \phi_{x}^{2}+n^{2}-\frac{1}{2} n^{-1 / 2}\left(n^{1 / 2}\right)_{x x}+V(X)=0,
\end{aligned}
$$

which are similar to the ones that have been employed to discuss the crossover from TG to BEC regime [17,18,32]. The ground state of the system may be obtained upon assuming that the atomic velocity $v \equiv \phi_{x}=0$ (i.e., no flow in the system) and $\phi_{t}=-\mu_{0}$ (dimensionless chemical potential). Then, as Eq. (3) implies that $n=n_{0}$ is time-independent in the ground state, we assume that $n_{0}=n_{0}(X)$. As the quantum pressure term in Eq. (4) is of order $O\left(\epsilon^{3}\right)$, to leading order in $\epsilon$ [to $\left.O(1)\right]$, we obtain

$$
n_{0}(X)=\sqrt{\mu_{0}-V(X)},
$$

in the region where $\mu_{0}>V(X)$, and $n_{0}=0$ outside. Equation (5) gives the density profile in the so-called Thomas-Fermi (TF) approximation [23]. It follows from Eq. (5) that in the case of the harmonic trap the axial size of the gas is $2 L$, where $L=\sqrt{2 \mu_{0}} / \Omega$. We now consider the propagation of small-amplitude linear excitations (e.g., sound waves) of the ground state, by seeking solutions of Eqs. (3)-(4) of the form,

$$
n=n_{0}(X)+\epsilon \tilde{n}(x, t), \quad \phi=-\mu_{0} t+\epsilon \tilde{\phi}(x, t),
$$

where the functions $\tilde{n}$ and $\tilde{\phi}$ describe the linear excitations. Substituting Eqs. (6) in Eqs. (3)-(4), to order
$O(1)$ we recover the $\mathrm{TF}$ approximation, while to order $O(\epsilon)$ we obtain the following system of linear equations,

$$
\tilde{n}_{t}+n_{0} \tilde{\phi}_{x x}=0, \quad \tilde{\phi}_{t}+2 n_{0} \tilde{n}-\frac{1}{4 n_{0}} \tilde{n}_{x x}=0 .
$$

The dispersion relation of the excitations can easily be obtained, upon considering plane-wave solutions of Eqs. (7) of the form $(\tilde{n}, \tilde{\phi})=\left(\tilde{n}_{0}, \tilde{\phi}_{0}\right) \exp [i(k x-\omega t)]$, where $\tilde{n}_{0}$ and $\tilde{\phi}_{0}$ are independent of $x$ and $t$ (but may depend on $X$ ). This way, we readily obtain the equation,

$$
\omega= \pm \sqrt{2 n_{0}^{2}(X) k^{2}+\frac{1}{4} k^{4}}
$$

which is a Bogoliubov-type excitation spectrum, but with the excitation frequency $\omega$ being a function of the slow variable $X$. The speed of sound is local and is given by

$$
c_{S}=s \sqrt{2} n_{0}(X)
$$

where $s=\operatorname{sign}\left(c_{S}\right)= \pm 1$, i.e., the sound may propagate in two opposite directions. Note that the local character of the speed of sound is due to the presence of the external potential, which bears resemblance to the sound propagation in slowly-varying nonuniform media [33].

We now employ the reductive perturbation method $[34,35]$ (see also [3] for a relevant study in BECs) to examine the evolution of the nonlinear excitations (e.g., solitons), of the ground state. As Eqs. (3)-(4) are inhomogeneous, we introduce a new slow time-variable, $T=\epsilon^{1 / 2}\left(t-\int^{x} C^{-1}\left(x^{\prime}\right) d x^{\prime}\right)$, where $C$ is the (local) velocity of the nonlinear excitations, to be determined in a self-consistent manner. Also, we introduce the following asymptotic expansions for the functions $n$ and $\phi$,

$$
\begin{aligned}
& n=n_{0}(X)+\epsilon n_{1}(X, T)+\epsilon^{2} n_{2}(X, T)+\cdots, \\
& \phi=-\mu_{0} t+\epsilon^{1 / 2} \phi_{1}(X, T)+\epsilon^{3 / 2} \phi_{2}(X, T)+\cdots .
\end{aligned}
$$

Substituting the expansions (10) into Eqs. (3)-(4), we obtain the following results: First, to order $O(1)$, Eq. (4) leads to the TF approximation [see Eq. (5)]. Then, to orders $O(\epsilon)$ and $O\left(\epsilon^{3 / 2}\right)$, Eqs. (4) and (3) respectively lead to the following system of linear equations:

$$
\phi_{1 T}+2 n_{0} n_{1}=0, \quad n_{1 T}+C^{-2} n_{0} \phi_{1 T T}=0 .
$$

The compatibility condition of Eqs. (11) is the equation $1-2 n_{0}^{2} C^{-2}=0$, which determines the unknown velocity $C$, which, actually, is the same as the speed of sound, i.e., $C \equiv c_{S}$ [c.f. Eq. (9)]. On the other hand, Eqs. (11) lead to the following equation,

$$
\phi_{1}(X, T)=-2 n_{0}(X) \int^{T} n_{1}\left(X, T^{\prime}\right) d T^{\prime},
$$

connecting the amplitude $n_{1}$ and the phase $\phi_{1}$.

Proceeding to the next order, namely to order $O\left(\epsilon^{2}\right)$ and to order $O\left(\epsilon^{5 / 2}\right)$, Eqs. (4) and (3) respectively read: 


$$
\begin{aligned}
& \phi_{2 T}+2 n_{0} n_{2}=-n_{1}^{2}-\frac{1}{2} C^{-2} \phi_{1 T}^{2}+\frac{1}{4} C^{-2} n_{0}^{-1} n_{1 T T}, \\
& n_{2 T}+C^{-2} n_{0} \phi_{2 T T}=-C^{-2}\left(n_{1} \phi_{1 T}\right)_{T} \\
& +C^{-1}\left(n_{0 X} \phi_{1 T}+2 n_{0} \phi_{1 X T}\right)+\frac{d C^{-1}}{d X} n_{0} \phi_{1 T} .
\end{aligned}
$$

The compatibility condition of Eqs. (13) and (14) yields the equation $1-2 n_{0}^{2} C^{-2}=0$ for the velocity $C$, along with the following nonlinear evolution equation for $n_{1}$,

$$
n_{1 X}-\frac{C}{n_{0}^{3}} n_{1} n_{1 T}+\frac{1}{32 n_{0}^{4} C} n_{1 T T T}=-\frac{d}{d X}\left(\ln n_{0}\right) n_{1},
$$

which is obtained upon also using Eq. (12). Equation (15) has the form of a $\mathrm{KdV}$ equation with variable coefficients, which has been used to describe shallow waterwaves over variable depth, or ion-acoustic solitons in inhomogeneous plasmas [35]. The inhomogeneity-induced dynamics and dissipation of the $\mathrm{KdV}$ solitons has been studied analytically [36,37]. Below, we will employ these results to analyze the coherent evolution and dissipation of dark solitons in an atomic gas in the TG limit.

Introducing the transformations $\chi=\int\left(32 n_{0}^{4} C\right)^{-1} d X$, $\tau=T$ and $n_{1}=\left(3 / 32 n_{0}^{3}\right) v(\chi, \tau)$, we put Eq. (15) into the form,

$$
v_{\chi}-6 v v_{\tau}+v_{\tau \tau \tau}=\lambda(\chi) v
$$

where $\lambda(\chi) \equiv 2\left(\ln n_{0}\right)_{\chi}$. In the case $\lambda=0$, i.e., for a homogeneous gas with $n_{0}(X)=n_{\mathrm{o}}=$ const., Eq. (16) is the completely integrable $\mathrm{KdV}$ equation, which possesses a single-soliton solution of the following form [38],

$$
v=-2 \kappa^{2} \operatorname{sech}^{2} Z, \quad Z=\kappa[\tau-\zeta(\chi)],
$$

where $\zeta(\chi)=4 \kappa^{2} \chi+\zeta_{0}$ is the soliton center (with $d \zeta / d \chi=4 \kappa^{2}$ being the soliton velocity in the $\tau$ - $\chi$ reference frame), while $\kappa$ and $\zeta_{0}$ are arbitrary constants presenting the soliton's amplitude (as well as inverse temporal width) and initial position respectively. Apparently, Eq. (17) describes a density notch on the backround density $n_{\mathrm{o}}$, with a phase jump across it [see Eq. (12), which implies that $\left.\phi_{1} \sim \tanh Z\right]$ and, thus, it represents an approximate dark soliton solution of Eq. (2).

In the general case of the inhomogeneous gas (i.e., in the presence of the trapping potential), soliton dynamics can still be studied analytically, provided that the right-hand side of Eq. (16) can be treated as a small perturbation. As $\lambda(\chi)$ is directly proportional to the density gradient, such a perturbative study is relevant in regions of small density gradients, which is consistent with the use of the local density approximation. In this case, employing the perturbation theory for solitons [39], we express the solution of Eq. (16) as,

$$
v=v_{S}+v_{R}
$$

where $v_{S}$ is the soliton part, which has the same functional form as in the unperturbed homogeneous case (c.f.
Eq. (17)), but with the soliton parameters $\kappa$ and $\zeta$ being now unknown functions of $\chi$. The contribution $v_{R}$, being of the same order of smallness as $\lambda$, denotes the radiation part of the solution (i.e., the sound profile) due to the effect of axial inhomogeneity. Following [37], we first derive the following evolution equations for the soliton's amplitude $\kappa(\chi)$ and center $\zeta(\chi)$,

$$
\frac{d \kappa}{d \chi}=\frac{2}{3} \kappa \lambda, \quad \frac{d \zeta}{d \chi}=4 \kappa^{2}+\frac{\lambda}{3 \kappa} .
$$

These equations can be solved analytically and the results, expressed in terms of the slow variable $X$, read:

$$
\begin{aligned}
& \kappa(X)=\kappa(0)\left(\frac{n_{0}(X)}{n_{0}(0)}\right)^{4 / 3}, \\
& \zeta(X)=\frac{\kappa^{2}(0)}{8 \sqrt{2} s n_{0}^{8 / 3}(0)} \int_{0}^{X} n_{0}^{-7 / 3}\left(X^{\prime}\right) d X^{\prime} \\
& +\frac{1}{3 \kappa(0)}\left[1-3\left(\frac{n_{0}(X)}{n_{0}(0)}\right)^{-1 / 3}\right],
\end{aligned}
$$

where $\kappa(0)$ and $n_{0}(0)$ are respectively the soliton amplitude and density at $X=0$, while $s= \pm 1$ represents the two possible directions of the soliton propagation. Additionally, we find the following approximate (for $|Z| \gg 1$ ) expression for the radiation part of the solution (i.e., sound wave emitted by the soliton),

$v_{R} \approx-\frac{32 \sqrt{2} s n_{0}^{4 / 3}(0)}{3 \kappa(0)} n_{0}^{8 / 3}(X) \frac{d n_{0}(X)}{d X}(1-\tanh Z)$

where $Z=\kappa(X)[\tau-\zeta(X)]$ is the soliton phase, with $\kappa(X)$ and $\zeta(X)$ given by Eqs. (20)-(21).

Based on the above results for the evolution of the soliton parameters, we will now show that the dark soliton will display an oscillatory motion in the harmonic trap $V(X)=(1 / 2) \Omega^{2} X^{2}$. This can be done upon deriving the phase of the soliton, which, to order $O\left(\epsilon^{3 / 2}\right)$, reads

$$
\begin{aligned}
& Z=\epsilon^{1 / 2} \mu_{0}^{2 / 3} \kappa(0)\left[1-\frac{4}{3}\left(\frac{X}{L}\right)^{2}\right] \\
& \times\left[t-\int \frac{d X}{C}-\epsilon \frac{\kappa^{2}(0) \mu_{0}^{-2 / 3}}{8 s \Omega}\left(\frac{X}{L}\right)\right]
\end{aligned}
$$

(recall that $L=\sqrt{2 \mu_{0}} / \Omega$ defines the axial size of the gas). Then, looking along the characteristic lines of soliton motion, it is easy to show that the position of the soliton satisfies the following equation of motion,

$$
\frac{d X}{d t}=\frac{8 \sqrt{2} s \Omega L n_{0}(X)}{8 \Omega L+\epsilon \sqrt{2} \kappa^{2}(0) \mu_{0}^{-2 / 3} n_{0}(X)} .
$$

For sufficiently small $\epsilon$ the second term in the denominator can be neglected and the separable resulting equation can readily be integrated. In particular, taking into regard Eq. (5) for a parabolic trap, we find that 


$$
X=L \sin (\Omega t),
$$

which demonstrates that a shallow dark soliton in the TG limit oscillates at the trap frequency.

In conclusion, we have developed a systematic analytical approach, based on a reductive perturbation method, to study the linear and nonlinear excitations of a Bose gas of impenetrable bosons (i.e., in the Tonks-Girardeau limit). We have recovered the Bogoliubov spectrum of linear excitations, with excitation frequencies (and speed of sound) varying slowly along the axial direction. Additionally, we have shown that shallow dark solitons can be described by an effective Korteweg-de Vries equation with variable coefficients. We have found analytical expressions for the inhomogeneity-induced evolution of the soliton parameters (amplitude, width, position, velocity) and the profile of the sound emitted by the soliton.

Our results are based on the quintic nonlinear Schrödinger equation $[20,21,24-29]$, which is expected to be valid for weak density modulations. This approach enables analytical results to be obtained, and the oscillation frequency of shallow solitons is thus found to be identical to the one obtained via numerical simulations based on the Bose-Fermi mapping [19]. This result seems to additionally justify a posteriori the use of Eq. (1) for shallow solitons. In particular, our work demonstrates the validity of the reductive perturbation method for dynamics of shallow solitons in the Tonks-Girardeau (TG) regime, while an earlier study [3] confirmed its usefulness in the opposite regime of Bose-Einstein condensation (BEC). The crossover between these two regimes [17] is important, and diagnostics (e.g. [32]) for the degree of "impenetrability" of a trapped one-dimensional Bose gas are required for interpreting current and future experiments [12]. The presented technique paves the way for investigating the crossover in the oscillation frequency of shallow dark solitons [ranging from $\Omega / \sqrt{2}$ in the BEC regime, to $\Omega$ in the TG regime ( $\Omega$ is the trap frequency)], upon suitably generalizing the nonlinearity to a smooth function interpolating between these two regimes $[17,30]$.

We acknowledge fruitful discussions with J. Brand and L. Santos. This work was supported by the Eppley Foundation for Research, NSF-DMS-0204585 and NSFCAREER (PGK).

[1] S. Burger et al., Phys. Rev. Lett. 83, 5198 (1999); J. Denschlag et al. Science 287, 97 (2000); B.P. Anderson et al., Phys. Rev. Lett. 86, 2926 (2001); Z. Dutton et al., Science 293, 663 (2001).

[2] Th. Busch and J.R. Anglin, Phys. Rev. Lett. 84, 2298 (2000).

[3] G. Huang et al., Phys. Rev. A 65, 053605 (2002).

[4] D.J. Frantzeskakis et al. Phys. Rev. A 66, 053608 (2002).
[5] P.O. Fedichev et al., Phys. Rev. A 60, 3220 (1999); A. Muryshev et al., Phys. Rev. Lett. 89, 110401 (2002).

[6] D.L. Feder et al., Phys. Rev. A 62, 053606 (2000); J. Brand and W.P. Reinhardt, Phys. Rev. A 65, 043612 (2002).

[7] N.G. Parker et al., Phys. Rev. Lett. 90, 220401 (2003).

[8] N.G. Parker et al., J. Phys. B 36, 2891 (2003).

[9] G. Theocharis et al., Phys. Rev. Lett. 90, 120403 (2003).

[10] M. Olshanii, Phys. Rev. Lett. 81, 938 (1998).

[11] L. Tonks, Phys. Rev. 50, 955 (1936); M. Girardeau, J. Math. Phys. (N.Y.) 1, 516 (1960).

[12] H. Moritz et al., Phys. Rev. Lett. 91, 250402 (2003); J. Reichel and J.H. Thywissen, cond-mat/0310330 (2003); B. Laburthe et al., cond-mat/0312003 (2003).

[13] E.H. Lieb and W. Liniger, Phys. Rev. 130, 1605 (1963).

[14] M.D. Girardeau and E.M. Wright, Phys. Rev. Lett. 84, 5691 (2000).

[15] M.D. Girardeau, Phys. Rev. Lett. 91, 040401 (2003).

[16] D.S. Petrov et al., Phys. Rev. Lett. 85, 3745 (2000).

[17] V. Dunjko et al., Phys. Rev. Lett. 86, 5413 (2001).

[18] P. Öhberg and L. Santos, Phys. Rev. Lett. 89, 240402 (2002).

[19] Th. Busch and G. Huyet, J. Phys. B 36, 2553 (2003).

[20] E.B. Kolomeisky et al., Phys. Rev. Lett. 85, 1146 (2000).

[21] R.K. Badhuri et al J. Phys. A 34, 6553 (2001).

[22] B. Damski et al, J. Phys. B 35, L153 (2002); T. Karpiuk et al, J. Phys. B 35, L315 (2002).

[23] F. Dalfovo et al. Rev. Mod. Phys. 71, 463 (1999).

[24] E.B. Kolomeisky and J.P. Straley, Phys. Rev. B 46, 11749 (1992);

[25] B. Tanatar, Europhys. Lett. 51, 261 (2000).

[26] Y.E. Kim and A.L. Zubarev, Phys. Rev. A 67, 015602 (2003).

[27] M.D. Lee et al., Phys. Rev. A 65, 043617 (2002); M.D. Lee et al., cond-mat/0305416 (2003).

[28] E.H. Lieb et al., Phys. Rev. Lett. 91, 150401 (2003).

[29] A. Minguzzi et al., Phys. Rev. A 64, 033605 (2001).

[30] J. Brand, cond-mat/0311206 (2003).

[31] M.D. Girardeau and E.M. Wright, Phys. Rev. Lett. 84, 5239 (2000).

[32] C. Menotti and S. Stringari, Phys. Rev. A 66, 043610 (2002).

[33] L.D. Landau and E.M. Lifshitz, Fluid Mechanics (Pergamon, New York, 1959).

[34] T. Taniuti, Prog. Theor. Phys. Suppl. 55, 1 (1974).

[35] N. Asano, Prog. Theor. Phys. Suppl. 55, 52 (1974).

[36] K. Ko and H.H. Kuehl, Phys. Rev. Lett. 40, 233 (1978); K. Ko and H.H. Kuehl, Phys. Fluids 23, 834 (1980).

[37] V.I. Karpman and E.M. Maslov, Phys. Fluids 25, 1686 (1982).

[38] M.J. Ablowitz and P.A. Clarkson, Solitons, Nonlinear Evolution Equations and Inverse Scattering (Cambridge University Press, Cambridge, England, 1991).

[39] V.I. Karpman and E.M. Maslov, Zh. Eksp. Teor. Fiz. 75, 504 (1978) [Sov. Phys.-JETP 48, 252 (1978)]; V.I. Karpman, Phys. Scripta 20, 462 (1979). 